\title{
Viel hilft viel?
}

\section{Im Gegensatz zur englischen Aromatherapie werden ätherische Öle in der FRANZÖSISCHEN AROMATHERAPIE hochdosiert. Ist die fran- zösische Aromatherapie Heilpraktikern dennoch zu empfehlen?}

Eliane Zimmermann

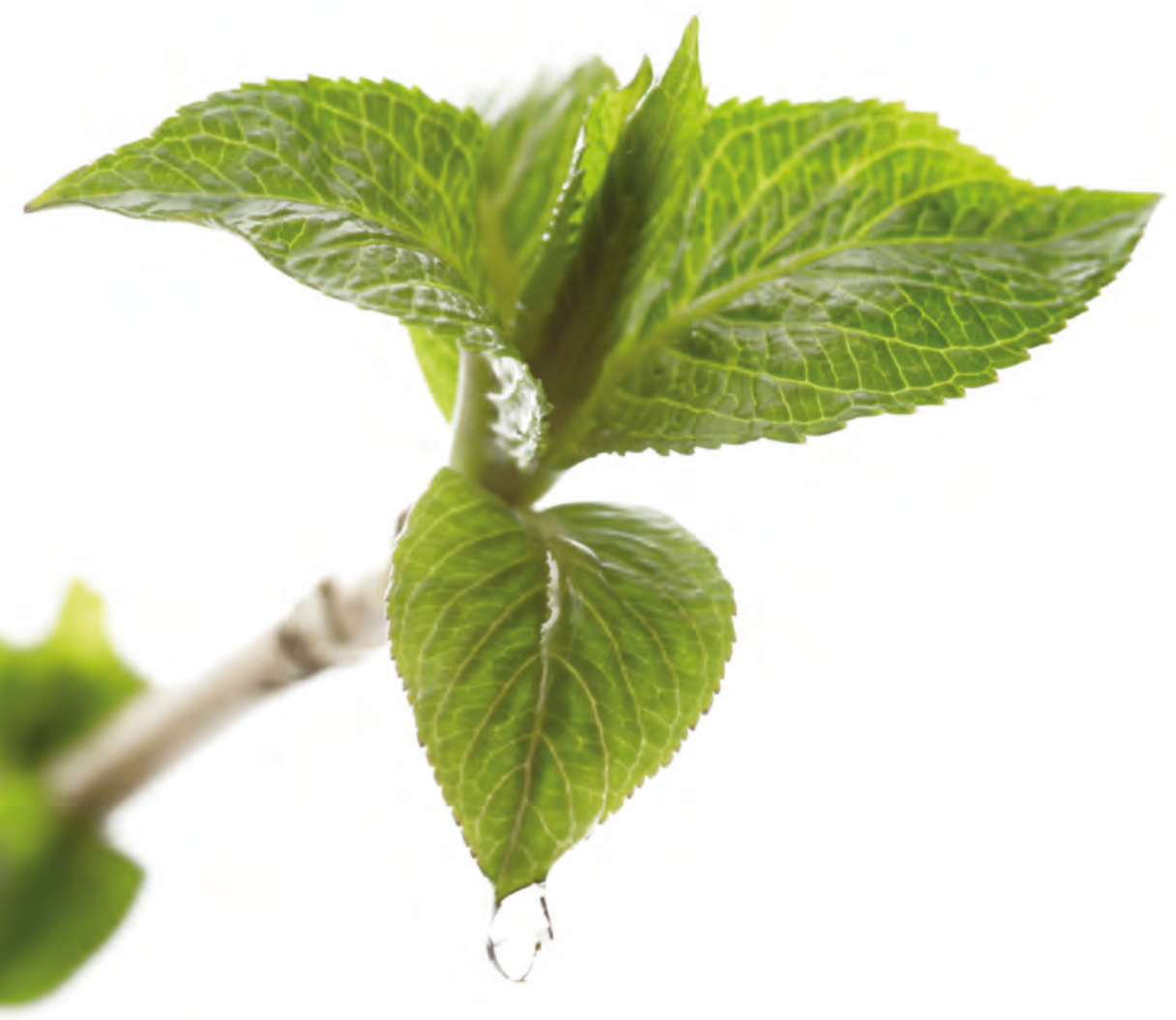

IN DER AROMATHERAPIE und -pflege sei es in Praxen, Kliniken, Pflegeheimen oder Hospizen - haben sich Anwendungen im 2\%-Bereich etabliert. Das heißt, das Verhältnis zwischen dem ätherischen Öl und dem Trägeröl beträgt ca. 2:100. Dieser Richtwert stammt aus der sogenannten englischen Aromatherapie. Er hat sich als höchst effektiv bewährt und garantiert gleichzeitig maximale Verträglichkeit bei den allermeisten Menschen. Daher arbeiten auch die Aromatherapeuten in Deutschland in der Regel mit Verdünnungen um die $2 \%$. Bei sehr sensiblen Patienten wie Kleinkindern und sehr alten sowie sterbenden Menschen wirken bereits Mischungen unter $1 \%$. Deshalb sollte man bei ihnen nicht höher dosieren. Weitere Abweichungen vom $2 \%$-Richtwert ergeben sich aus Faktoren wie Alter, Art der Anwendung, Indikationen etc. (siehe Tabelle 2).

\section{Ist die französische Aromatherapie besser?}

In den letzten Jahren werden immer mehr Rufe nach der französischen Aromatherapie laut. Französische Aromatherapie heißt im Klartext: Man wendet ätherische Öle nicht nur wie in der englischen Aromatherapie üblich als Raumduft, für Inhalationen oder topisch an, sondern nimmt sie auch oral ein. Auch rektale und vaginale Behandlungen sind Standard. Hinzu kommt - und das ist womöglich das wichtigste Unterscheidungsmerkmal zur englischen Aromatherapie: Man verwendet hochprozentige Verdünnungen beziehungsweise verdünnt die Öle gar nicht.

Insbesondere Verkaufsrepräsentanten einiger Anbieter von ätherischen Ölen behaupten, die französische Aromatherapie lehre die einzig richtige Art der Anwendung. In jüngster Vergangenheit 
wird auch immer wieder behauptet, dass Vertreter der englischen Aromatherapie mit ihren niedrigen Dosierungen völlig ineffektiv arbeiten würden. „Honi soit qui mal y pense“ oder: „Ein Schelm, wer Böses dabei denkt." Steht bei diesen Behauptungen womöglich eher der Umsatz im Vordergrund als die Sorge um eine effektive Therapie? Kommt der hochdosierte Gebrauch von ätherischen Ölen in der französischen Aromatherapie einigen Herstellern ätherischer Öle gelegen?

\section{Aromatherapie in Frankreich: Ärzten vorbehalten}

In Frankreich ist die Aromatherapie ein streng ärztliches Verfahren. Das hat seine Gründe. Die französische Aroma-

- INFORMATION

\section{Wie entstand die französi- sche Aromatherapie?}

Wie der Name schon verrät, liegt die Wiege der französischen Aromatherapie in Frankreich. Als ihr Begründer gilt der Chemiker René-Maurice Gattefossé. Dieser hatte nach einem Laborunfall eine im wahrsten Sinne lebensrettende Begegnung mit Lavendelöl. Als er seinen brennenden Körper durch Rollen im Gras löschte, zog er sich einen durch das Bakterium Clostridium perfringens ausgelösten Gasbrand zu. Eine Erkrankung, die auch heute noch unter Therapie in etwa $50 \%$ der Fälle tödlich endet. Als Parfümeur war Gattefossé vertraut im Umgang mit ätherischen Ölen und wusste auch um die keimtötende Wirkung bestimmter Öle. Er schrieb über seine Erfahrung mit Lavendelöl. „Eine einzige Spülung mit Lavendelessenz brachte die ,Vergasung des Gewebes' zum Stillstand. Auf diese Behandlung folgte ein starker Schweißausbruch, die Heilung begann am nächsten Tag." [1]. Durch seinen Heilungserfolg angespornt, forschte Gattefossé weiter. Seine Erkenntnisse und sein 1937 erschienenes Buch „Aromathérapie - les huiles essentielles hormones végétales“ legten den Grundstein für die Aromatherapie.

\section{KURZ GEFASST

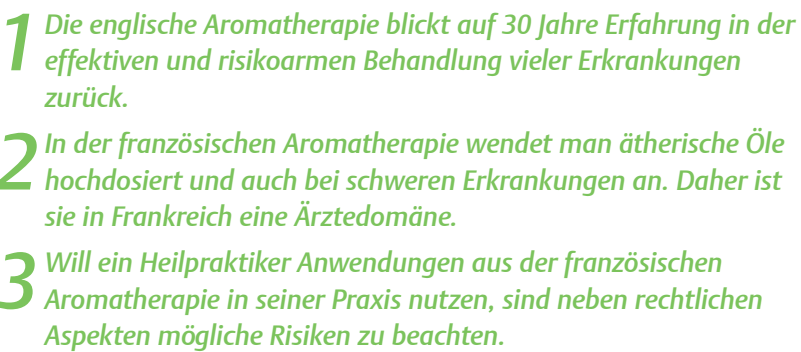

therapie setzt ätherische Öle auch bei schwereren und akuten Krankheiten inklusive Geschlechtskrankheiten ein wie:

- Pneumonie

" Prostatitis und Zystitis

- Psoriasis

- Otitis media

- virale Hepatitis

- Colitis ulcerosa

- tropische Infektionskrankheiten

- Kinderkrankheiten wie Varizellen und Pertussis

- Schlangenbisse

- vaginale Infektionen (Candida, Chlamydien)

Teilweise nutzt man auch in Deutschland als „toxisch“ deklarierte Öle: Zum Beispiel verabreicht man Muskatöl (Myristica fragrans) oder Wermutöl (Artemisia absinthium) kurzzeitig innerlich zur Behandlung von Darmparasiten. Flohminze (Mentha pulegium) empfiehlt man bei Keuchhusten von größeren Kindern und bei Bronchialasthma. Kalmuswurzelöl (Acorus calamus) wendet man bei Zystitis und Nierenstau an.

Man schreckt in der französischen Aromatherapie auch vor sehr hohen Dosierungen nicht zurück: Beispielsweise verordnete der französische Arzt Dr. Daniel Pénoël bei Morbus Crohn Kunzeaöl (Kunzea ambigua) zur oralen Einnahme, $3 \times$ tgl. 6 Tr. in Basisöl [2]. Das sind 18 Tropfen täglich.

In manchen Fällen verwendet die französische Aromatherapie auch kaum verdünnte oder unverdünnte Öle. Daher sind in Frankreich etliche ätherische Öle, die man im deutschsprachigen Raum bei jedem Anbieter findet, rezeptpflichtig oder dürfen nur verdünnt verkauft werden. Dazu gehören Salbei (Salvia officinalis) und Ysop (Hyssopus officinalis). Denn sie enthalten die potenziell toxischen Monoterpenketone Thujon und Pinocamphon. Diese wirken bei niedriger Dosierung zwar neurotonisch (konzentrationsfördernd), bei Überdosierung diese fällt individuell unterschiedlich aus - jedoch neurotoxisch.

Infektionen des Atemtraktes behandelt die französische Aromatherapie mit recht hochdosierten Aroma-Suppositorien, sodass die Wirkstoffe die Atemwege ohne Beteiligung des Leberstoffwechsels erreichen. Es sind beispielsweise ätherische Öle, die reich an sekretolytischem 1,8-Cineol (zum Beispiel Cinnamomum camphora CT 1,8-Cineol) oder an antibakteriellem Thymol (zum Beispiel Thymus vulgaris CT Thymol) sind. Eine erfahrene Aromatherapeutin in Frankreich empfiehlt bei nahender Grippe: Thymian CT Thymol 20\%, Eucalyptus globulus $30 \%$, Teebaum 20\%, Schwarzkiefer 20\% und Pfefferminze $10 \%$ in einem 175 -mgZäpfchen. Für Kinder empfiehlt der belgische Apotheker Dominique Baudoux $80 \mathrm{mg}$ ätherisches Öl pro Zäpfchen bei einer Dosis von $3 \times$ tgl. 1 Zäpfchen [3].

Auch Einreibungen mit kaum verdünnten ätherischen Ölen oder Inhalationen mit hochdosierten ätherischen Ölen sind in der französischen Aromatherapie üblich: Dr. Daniel Pénoël empfiehlt Menschen mit niedrigem Blutdruck, frühmorgens die Nebennierengegend kräftig mit einigen Tropfen unverdünntem Balsamtannenöl (Abies balsamea) einzureiben. So komme der Kreis- 
Abb. 1 Zäpfchen: Keine ungewöhnliche Anwendungsform in der französischen Aromatherapie. Foto: (C) Fotolia/Richard Villalon

lauf gut in Schwung. Auch empfiehlt er bei Sinusitis, kurze Inhalationsstöße mit unverdünntem ätherischen Öl aus einem Kaltzerstäuber tief einzuatmen. Die sekretolytische Wirkung kann somit nach kürzester Zeit eintreten. Für diese Anwendung kommen verschiedene Öle infrage, zum Beispiel Cajeput [4].

\section{Eignet sich die französische Aromatherapie auch für die Heillpraktikerpraxis?}

Fakt ist, dass die englische Aromatherapie auf mehr als 30 Jahre Erfahrung in der erfolgreichen Behandlung verschiedenster Erkrankungen von allergischem Schnupfen bis Zystitis zurückblickt. Wer in der englischen Aromatherapie geschult ist und sie in seiner Praxis einsetzt, ist gut beraten, dies weiterhin zu tun. Denn sie ist, wie bereits erwähnt, nicht nur effektiv, sondern auch risikoarm.

Die französische Aromatherapie hat natürlich auch ihre Vorteile: Setzen medizinisch geschulte Personen ätherische Öle im Sinne der französischen Aromatherapie ein, können diese oftmals Antibiotika, Schmerzmittel oder Kortisonpräparate ersetzen oder zumindest einsparen helfen. Dies ist mit der englischen Aromatherapie eher nicht möglich oder nur im Rahmen einer langwierigen Behandlung.

Voraussetzung für die Anwendung der französischen Aromatherapie ist eine einschlägige Ausbildung und eine aus- führliche Lektüre entsprechender Literatur, die es bisher größtenteils nur auf Französisch gibt. Nicht anders als in der englischen Aromatherapie sind auf der einen Seite gute Kenntnisse der menschlichen Physiologie wichtig. Auf der anderen Seite muss man die Zusammensetzung aller verwendeten ätherischen Öle ausreichend kennen, sodass man Unverträglichkeiten, insbesondere bei sensiblen Patientengruppen, und Wechselwirkungen mit Medikamenten ausschließen kann. Auch die recht schnell eintretende Oxidation bestimmter Öle (zum Beispiel Teebaumöl, Zitrusöle, Nadelöle, zitronig duftende Öle) muss man sicher einschätzen können, sodass keine unnötigen Reizungen verursacht werden. Darüber hinaus ist detailliertes Wissen zur Haltbarkeit, zur Lagerung sowie zu Handhabung verschiedener Öle Pflicht.

\section{Rechtliche Aspekte und Risiken}

Für die Aromapflege, wie sie zunehmend in deutschen Krankenhäusern und Pflegeheimen eingesetzt wird, sind Verdünnungen von maximal 3\% vorgeschrieben. Für Angehörige von Heilberufen gibt es keine gesetzlichen Vorschriften. Allerdings sind viele ätherische Öle in Deutschland, die für den aromatherapeutischen Einsatz infrage kommen, als
Kosmetika für die Aromapflege deklariert und somit nur als solche zugelassen. Entsprechend niedrig prozentig sind die Dosierungsempfehlungen.

Im Zuge der Therapiefreiheit und im Rahmen der Gesetzeslage (AMG) kann ein Heilpraktiker hochdosierte Anwendungen der französischen Aromatherapie durchführen, wenn er in seiner Praxis aus ätherischen Ölen und einer Trägerlösung für einen individuellen Patienten ein Arzneimittel herstellt und dieses

" unmittelbar,

" ohne Lagerung und Abgabe an den Patienten

- direkt am Patienten in seiner Praxis anwendet.

Es besteht dann Anzeigepflicht nach $\S 67$ Abs. 2 AMG. Diesen Aufwand muss der Heilpraktiker auf sich nehmen. Salbenanwendungen sind kritisch zu betrachten, denn sie sind meist für mehrmalige Anwendungen durch den Patienten selbst gedacht. Die Salbe müsste also an den Patienten abgegeben werden, was in dem Fall nicht zulässig wäre.

Eine heikle Frage der Risiko-NutzenAbschätzung ist, ob man zu Maßnahmen wie Zäpfchen greifen will. Denn diese können unter Verwendung von potenziell reizenden Ölen wie Pfefferminzöl und Teebaumöl oder potenziell toxischen Ölen wie Salbeiöl oder Ysopöl in höheren Dosierungen gesundheitliche Risiken bergen.

\section{TABELLE 1}

\section{Die englische und französische Aromatherapie im Vergleich}

\begin{tabular}{|c|c|}
\hline englische Aromatherapie & französische Aromatherapie \\
\hline niedrige Dosierungen zwischen 0,5 und 3\% & $\begin{array}{l}\text { hohe Dosierungen bis zu unverdünnten } \\
\text { Ölen }\end{array}$ \\
\hline $\begin{array}{l}\text { Ätherische Öle mit potenziell toxischen } \\
\text { Inhaltsstoffen werden nicht angewendet. }\end{array}$ & $\begin{array}{l}\text { Ätherische Öle mit potenziell toxischen In- } \\
\text { haltsstoffen können bei gezielten Indikatio- } \\
\text { nen eingesetzt werden. }\end{array}$ \\
\hline $\begin{array}{l}\text { im deutschen Sprachraum auch erlaubt im } \\
\text { Rahmen der Aromapflege und Kosmetik }\end{array}$ & $\begin{array}{l}\text { nur für Angehörige von Heilberufen geeig- } \\
\text { net }\end{array}$ \\
\hline $\begin{array}{l}\text { Orale, rektale und vaginale Anwendungen } \\
\text { sind im Pflegebereich nicht gestattet, in } \\
\text { der Therapie nicht üblich. }\end{array}$ & $\begin{array}{l}\text { Orale, rektale und vaginale Anwendungen } \\
\text { sind üblich. }\end{array}$ \\
\hline
\end{tabular}




\section{Ein gefährlicher Trend: Die Entgiftungstheorie}

Immer mehr Laienanwender in Deutschland berufen sich auf die ärztlich ausgeübte Aromatherapie in Frankreich. Sie behaupten, wie eingangs erwähnt, dass nur die französische Aromatherapie wirklich wirksam sei. In vielen Fällen haben sie sich nur rudimentäres Grundwissen in Schnell-Lehrgängen oder gar nur an Infoabenden angeeignet. Vielfach haben sie keinerlei Vorstellungen von physiologischen Vorgängen im menschlichen Körper oder der Pharmakologie der ätherischen Öle. Sie verwechseln zum Beispiel die recht harmlosen Monoterpenole mit den stark wirksamen Phenolen. Dennoch sind sie davon überzeugt: „viel hilft viel“, „ist ja alles Natur“, „Natur schadet nicht“, „laut der französischen Aromatherapie wird es ja auch so gemacht". Noch arglosere Kunden fallen darauf rein und erleiden in nicht wenigen Fällen schwere Hautreizungen. Unglaublich, aber wahr: Diese Verletzungen werden manchmal sogar mit dem Hinweis auf die französische Aromatherapie vorausgesagt. Die betreffende Person solle sich freuen, Rötungen und Blasen seien willkommene Zeichen der Entgiftung. Zu diesem Thema hat sich eine Mitarbeiterin Robert Tisserands, eines Spezialisten für den sicheren Umgang mit ätherischen Ölen, auf Englisch ausführlich geäußert: http://tisserandinstitute.org/essential-oils-and-the-detox-theory/

Ausgeschlossen für den Heilpraktiker sind natürlich auch alle Indikationen der französischen Aromatherapie, die er per Gesetz (zum Beispiel Infektionsschutzgesetz, IfSG) nicht behandeln darf. Darunter fallen zum Beispiel Geschlechtskrankheiten.

\section{Mein Fazit}

Ob hochprozentige Anwendung oder starke Verdünnung - beides hat seine Berechtigung. In der Aromatherapie sehr erfahrene Ärzte und Heilpraktiker könnten in vielen Fällen durch kurzzeitige hoch dosierte Anwendungen akute Beschwerden schnell lindern. Jedoch ist die französische Aromatherapie nicht so risikoarm wie die englische Aromatherapie. Wer also auf Nummer sicher gehen möchte und wer vor allem nicht gut in der französischen Aromatherapie ausgebildet ist, sollte auf die bei vielen Krankheiten sehr wirksame englische Aromatherapie setzen - und maximal mit Verdünnungen von $3 \%$ therapieren.
Dieser Artikel ist online zu finden: http://dx.doi.org/10.1055/s-0043-116164

Quellen

[1] Gattefossé RM, Tisserand R (Hrsg.). Gattefossés Aromatherapie. Der Klassiker der Aromatherapie. Aarau: AT; 1998

[2] Pénoël D. Vortrag auf Tagung von Forum Essenzia. Würzburg; Januar 2006

[3] Baudoux D. Les cahiers pratiques d'aromathérapie selon l'école française. Band 2. Brüssel: Amyris; 1998

[4] Pénoël D. Workshop bei Tagung von Veroma. Zürich; September 1995 
TABELLE 2

\section{Was müssen Sie bei welcher Verdünnung von ätherischen Ölen beachten?}

\begin{tabular}{|c|c|c|c|c|}
\hline $\begin{array}{l}\text { Verdünnung des } \\
\text { ätherischen Öles }\end{array}$ & 0,5-1 \% in Trägeröl & 1,5-3 \% in Trägeröl & $5-10$ \% in Trägeröl & pur, 1-3 Tropfen \\
\hline Alter & $\begin{array}{l}\text { - Säuglinge } \\
\text { - Kleinkinder bis circa } 5 \\
\text { Jahren } \\
\text { - verwirrte Menschen } \\
\text { - alte Menschen }\end{array}$ & $\begin{array}{l}\text { - } \text { stabile Kinder ab Schulein- } \\
\text { tritt 1,5\% } \\
\text { - } \text { Kinder ab } 12 \text { Jahren 3\% } \\
\text { - stabile Erwachsene }\end{array}$ & - stabile Erwachsene & $\begin{array}{l}\text { - Kinder ab } 3 \text { Jahren } \\
\text { - Erwachsene }\end{array}$ \\
\hline Anwendung & $\begin{array}{l}\text { - Raumbeduftung } \\
\text { - medizinische Massagen } \\
\text { - Inhalationen } \\
\text { - Bäder } \\
\text { - Waschungen } \\
\text { - Wickel und Auflagen }\end{array}$ & $\begin{array}{l}\text { - Raumbeduftung } \\
\text { - medizinische Massagen } \\
\text { - Inhalationen } \\
\text { - Bäder } \\
\text { - Waschungen } \\
\text { - Wickel und Auflagen }\end{array}$ & $\begin{array}{l}\text { - Sport- und medizinische } \\
\text { Massagen }\end{array}$ & - Direktanwendungen \\
\hline Kontraindikation & $\begin{array}{l}\text { keine Öle mit 1,8-Cineol } \\
\text { oder Menthol in Nasennä- } \\
\text { he }\end{array}$ & $\begin{array}{l}\text { - Vorsicht bei Kindern, auf } \\
\text { Schleimhäuten und bei } \\
\text { empfindlicher Haut } \\
\text { " relative Kontraindikation: } \\
\text { Salbei bei Epileptikern und } \\
\text { potenziell toxische Öle bei } \\
\text { empfindlichen Zielgrup- } \\
\text { pen, zum Beispiel komatö- } \\
\text { se Patienten }\end{array}$ & $\begin{array}{l}\text { in dieser Verdünnung folgen- } \\
\text { de Öle nicht anwenden: } \\
\text { - Bohnenkraut } \\
\text { - Thymian Thymol } \\
\text { - Gewürznelke } \\
\text { - Wintergrün } \\
\text { - Zimt }\end{array}$ & $\begin{array}{l}\text { In dieser Verdünnung folgen- } \\
\text { de Öle nicht anwenden: } \\
\text { " mit hohem Phenolanteil } \\
\text { (zum Beispiel Zimt und } \\
\text { Oregano) } \\
\text { - mit hohem Aldehydanteil } \\
\text { (zum Beispiel Litsea cube- } \\
\text { ba, Zitronenmyrte, Winter- } \\
\text { grün) }\end{array}$ \\
\hline
\end{tabular}

